\title{
Resilient Operation of Power Distribution Systems Using MPC-based Critical Service Restoration
}

Abinet Tesfaye Eseye (aeseye@nrel.gov), Bernard Knueven, Xiangyu Zhang, Matthew Reynolds, Wesley Jones Computational Science Center, NREL

The 2021 IEEE Green Technologies Conference (GreenTech)

April 7-9, 2021 


\section{Contents}

1 Background

2 Problem Statement \& Formulation

3 Results \& Discussion

4 Conclusion \& Future Work 


\section{Background}

- Incidence of extreme events (natural disasters, cyber and terrorist attacks) is rising globally due to the changes in weather conditions and socio-political threats.

- Standard definition and metrics for resilience are not available yet.

- Resilience - "the ability to anticipate and adapt to changing conditions and withstand and recover rapidly from disruptions," according to the U.S. PPD-2 1.

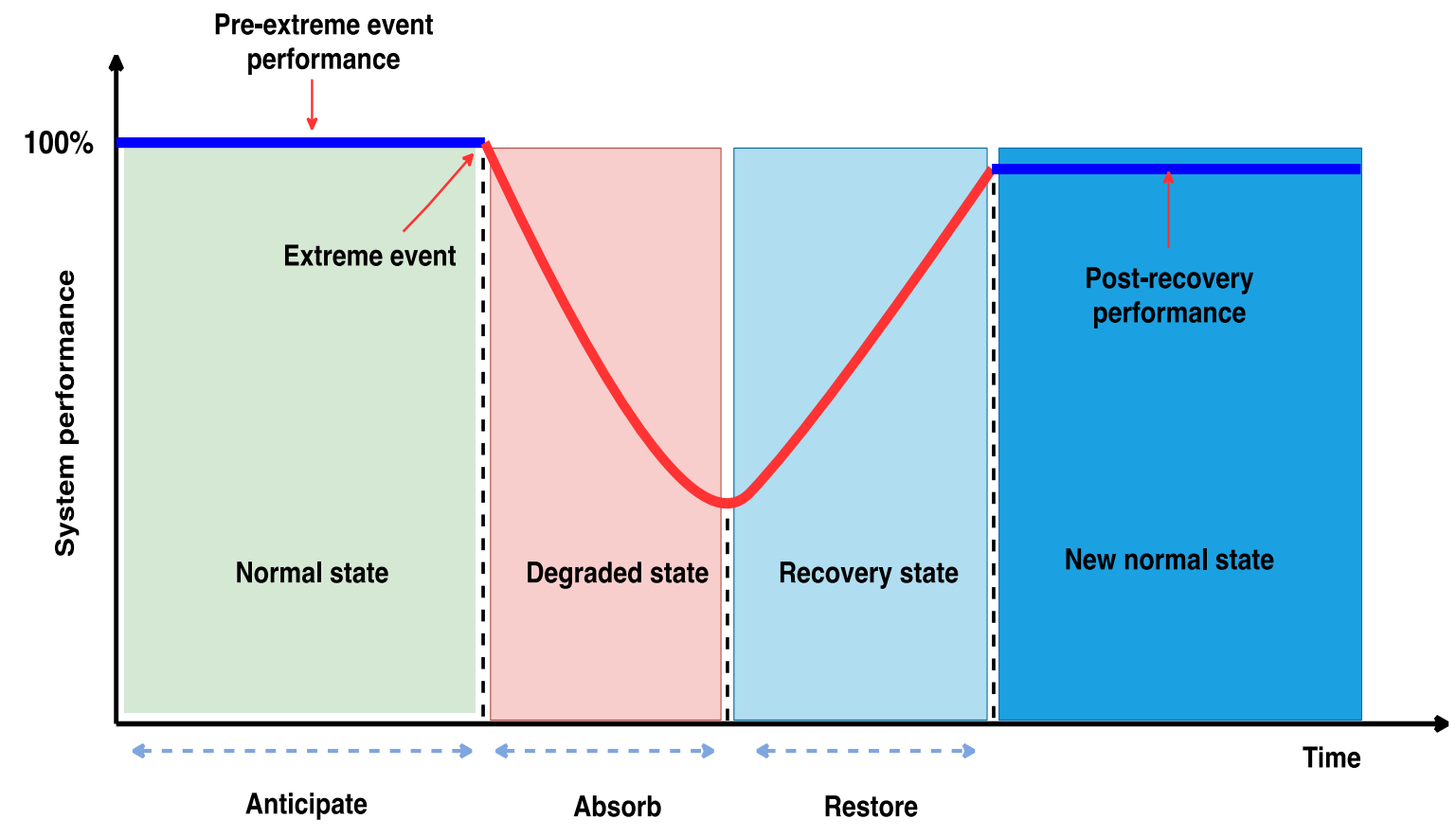

Resiliency curve

- $\quad$ Resilient power system should be able to anticipate, absorb and recover from extreme events. 


\section{Background}

- Data collected by power utilities show that about $90 \%$ of power outages in the U.S. initiated from distribution grids.

- Optimization-based prior research efforts on resilient operation of distribution systems can be categorized as:

- Single-step vs multi-step (look-ahead) formulations

- W/ network vs w/o network constraints

- W/ voltage vs w/o voltage bounds

- $\mathrm{W} /$ reserve vs w/o reserve products

- Load restoration is an important method to enhance the resilience of distribution grids during extreme events.

- Model predictive control (MPC) has become a popular control paradigm for sequential multiperiod control problems with variable/uncertain parameters and variables.

This paper devises OPF-driven, ramping reserve-augmented, MPC-based critical service restoration technique for resilient operation of active power distribution systems during extreme events. 


\section{Problem}

\section{Statement}

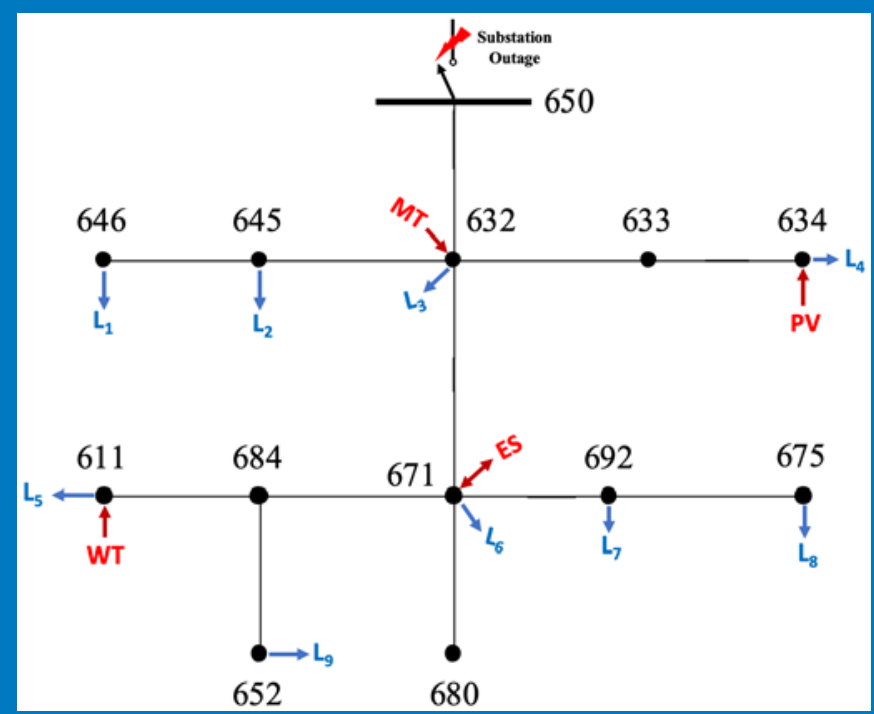

Distribution feeder hosting DERs

Notations:

MT - Microturbine

PV - Photovoltaic solar

WT - Wind turbine

ES - Energy storage

L - Electrical load

\section{Goal:}

We aim to maximize the amount of load restored following the incidence of an extreme event-triggered substation outage through the control of distributed energy resources (DERs) in the distribution feeder.

\section{Control Framework}

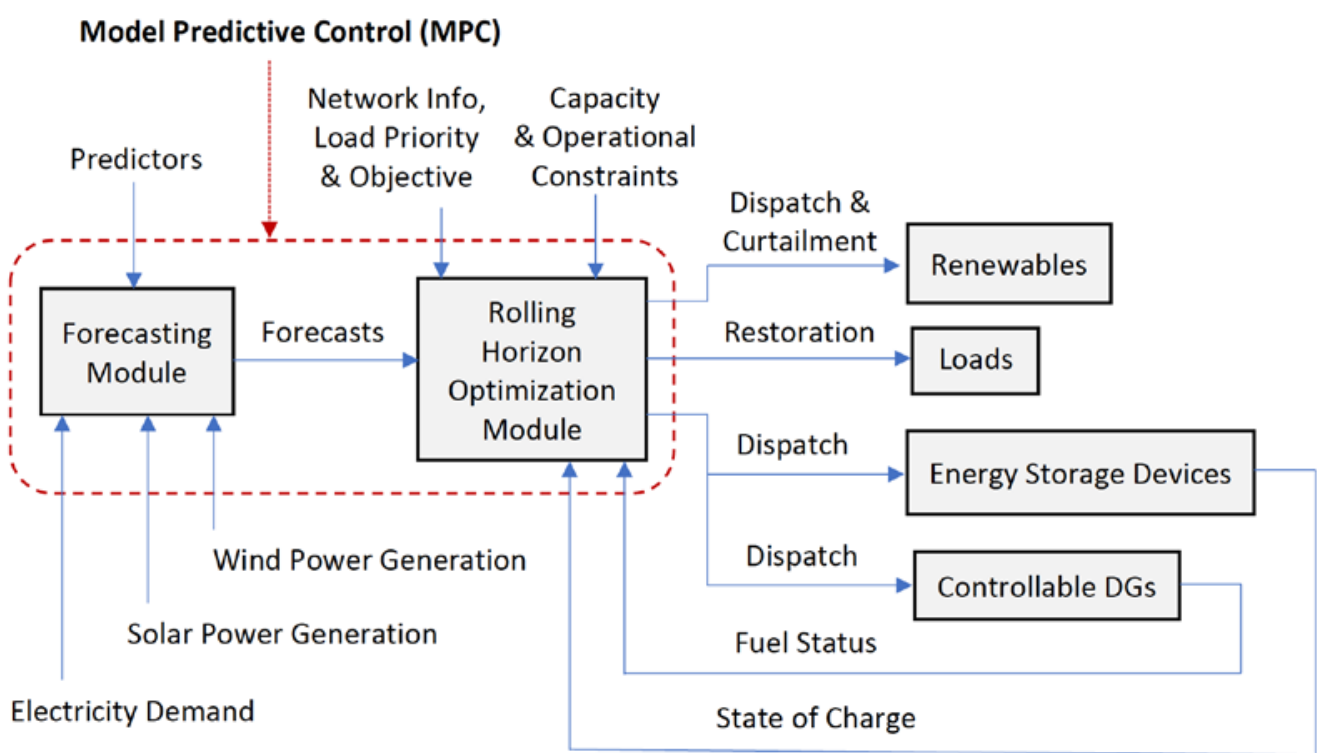




\section{Problem}

\section{Formulation}

\section{Objective Function}

Maximize C $=\left\{\sum_{i \in N} \sum_{t \in T} \frac{\omega_{i \cdot}\left(P_{i, t}^{l}+Q_{i, t}^{l}\right)}{\Gamma-P \text { Proritized restored load (active and reactive) }}\right.$

$-\psi_{P} \sum \sum \omega_{i} \cdot \max \left(\left(P_{i, t-1}^{l}-P_{i, t}^{l}\right), 0\right)$

$\sum_{i \in N} \sum_{t \in T\{\{1\}}$ Penalties for shedding prior

$-\psi_{Q} \sum_{i \in N} \sum_{t \in T \backslash\{1\}} \omega_{i} \cdot \max \left(\left(Q_{i, t-1}^{l}-Q_{i, t}^{l}\right), 0\right)>$ restored load (active and reactive)

$-\phi \sum_{t \in T} \max \left(\left(R_{t}-\sum_{i \in N}\left(P_{i, t}^{\text {graup }}+P_{i, t}^{e s, \text { raup }}\right)\right), 0\right)$ Penalty for not meeting

$\left.-\sum_{i \in N}\left(\alpha \sum_{t \in T} P_{i, t}^{w t, c u t}-\beta \sum_{t \in T} P_{i, t}^{p v, c u t}\right)\right\} . \Delta t \quad \begin{aligned} & \text { Penalty for renewable } \\ & \text { curtailment }\end{aligned}$

Notations:

$N$
$T$
$i$
$t$
$\omega$
$\psi_{P} / \psi_{Q}$
$\phi$
$\alpha / \beta$
$P_{i, t}^{l} / Q_{i, t}^{l}$
$R_{t}$
$P_{i, t}^{g, r a u p}$
$P_{i, t}^{e s, \text { raup }}$
$P_{i, t}^{\text {wt,cut }}$
$P_{i, t}^{\text {pv,cut }}$

number of nodes

control horizon (shrinks over time) node index

control interval (time) index

load priority weight

penalties for shedding prior restored active/reactive loads penalty for matching required and provided reserves penalties for wind and solar power curtailments active and reactive restored loads system-wide ramping (up) reserve requirement ramping reserve product of the dispatchable generator $g$ ramping reserve product of the energy storage device $e s$ wind power curtailed

PV power curtailed, at node $i$ and time $t$ 


\section{Problem}

\section{Formulation}

Constraints

$$
\left.\begin{array}{c}
P_{i j, t}=P_{j, t}^{l}-\left(P_{j, t}^{g}+P_{j, t}^{w t}-P_{j, t}^{w t, c u t}+P_{j, t}^{p v}-P_{j, t}^{p v, c u t}-P_{j, t}^{e s, c h}+P_{j, t}^{e s, d c h}\right) \\
+\sum_{k \in N} A_{j k} P_{j k, t}, \forall t \in T, \forall j \in N, i=r(j) \\
\begin{array}{c}
Q_{i j, t}=Q_{j, t}^{l}-\left(Q_{j, t}^{g}+Q_{j, t}^{w t}+Q_{j, t}^{p v}-Q_{j, t}^{e s, c h}+Q_{j, t}^{e s, d c h}\right) \\
+\sum_{k \in N} A_{j k} Q_{j k, t}, \forall t \in T, \forall j \in N, i=r(j) \\
V_{j, t}=V_{i, t}-2\left(r_{i j} P_{i j, t}+x_{i j} Q_{i j, t}\right), \forall t \in T, \forall j \in N, i=r(j)
\end{array}
\end{array}\right\}
$$

$$
\begin{aligned}
& v_{\min }^{2} \leq V_{j, t} \leq v_{\max }^{2} \\
& V_{j, t}(t)=\left(v_{j, t}\right)^{2} \\
& v_{j, t}=1, \forall t \in T, j=\text { slack bus } \\
& 0 \leq P_{j, t}^{g} \leq P_{j, \max }^{g} \\
& 0 \leq Q_{j, t}^{g} \leq Q_{j, \text { max }}^{g} \\
& 0 \leq P_{j, t}^{g, \text { raup }} \leq P_{j, \text { max }}^{g} \\
& P_{j, t}^{g}+P_{j, t}^{g, \text { raup }} \leq P_{j, \text { max }}^{g}
\end{aligned}
$$




\section{Problem \\ Formulation}

$$
\left.\begin{array}{c}
\sum_{t \in T}\left(P_{j, t}^{g}+P_{j, t}^{g, \text { raup }}\right) \Delta t \leq E_{j, \text { max }}^{g, p} \\
\sum_{t \in T} Q_{j, t}^{g} \Delta t \leq E_{j, \text { max }}^{g, q}
\end{array}\right\} \begin{gathered}
\text { Generator fuel (total energy } \\
\text { production) constraints }
\end{gathered}
$$

$Q_{j, t}^{l} /_{P_{j, t}^{l}}={ }^{l, d \text { demand }} /{ }_{P_{j, t}^{l, \text { demand }}}$

Power factor consistency constraint

Constraints

$$
\left.\begin{array}{c}
0 \leq P_{j, t}^{w t, c u t} \leq P_{j, t}^{w t} \\
0 \leq P_{j, t}^{p v, c u t} \leq P_{j, t}^{p v}
\end{array}\right\} \text { Renewable power curtailment limits }
$$




\section{Problem}

Formulation

Constraints

$$
\begin{aligned}
& 0 \leq P_{j, t}^{e s, c h} \leq b_{j, t}^{e s, c h} P_{j, \max }^{e s, c h} \\
& 0 \leq Q_{j, t}^{e s, c h} \leq b_{j, t}^{e s, c h} Q_{j, \max }^{e s, c h} \\
& 0 \leq P_{j, t}^{e s, d c h}+P_{j, t}^{e s, \text { raup }} \leq b_{j, t}^{e s, d c h} P_{j, \max }^{e s, d c h} \\
& 0 \leq Q_{j, t}^{e s, d c h} \leq b_{j, t}^{e s, d c h} Q_{j, \text { max }}^{e s, d c h} \\
& b_{j, t}^{e s, c h}+b_{j, t}^{e s, d c h}=1, b_{j, t}^{e s, c h}, b_{j, t}^{e s, d c h} \in\{0,1\}
\end{aligned}
$$$$
S O C_{j, \text { min }}^{e s} \leq S O C_{j, t}^{e s} \leq S O C_{j, \max }^{e s}
$$

Energy storage state of charge (SOC) limits

$$
\left.\begin{array}{l}
S O C_{j, t}^{e s}=S O C_{j, t-1}^{e s}+\left(\frac{\eta_{j}^{e s, c h} P_{j, t}^{e s, c h}}{C_{j}^{e s}}-\frac{P_{j, t}^{e s, d c h}}{\eta_{j}^{e s, d c h} C_{j}^{e s}}\right) \Delta t \\
S O C_{j, t}^{e s} \geq S O C_{j, \text { min }}^{e s}+P_{j, t}^{e s, r a u p} \Delta t
\end{array}\right\} \begin{gathered}
\text { Energy storage } \\
\text { SOC dynamics }
\end{gathered}
$$


$P_{j, t}^{l} \quad$ restored load (active power)

\section{Problem}

Formulation

Decision

Variables
$Q_{j, t}^{l}$

$P_{i j, t}$

$Q_{i j, t}$

$P_{j, t}^{g}$

$P_{j, t}^{g, r a u p}$

$Q_{j, t}^{g}$

$P_{j, t}^{e s}$

$Q_{j, t}^{e s, c h}$

$P_{j, t}^{e s, r a u p}$

$S O C_{j, t}^{e s}$

$b_{j, t}^{e s, c h} / b_{j, t}^{e s, d c h}$

$P_{j, t}^{w t, c u t}$

$P^{p v, c u t}$

$P_{j, t}$

$Q_{j, t}^{w t}$

$Q_{j, t}^{p v}$ restored load (active power)

line flow (active power)

line flow (reactive power)

generator active power

generator ramping reserve product

generator reactive power

storage active power

storage converter reactive power

storage ramping reserve product

storage state of charge

storage charge/discharge status indicator

wind power curtailment

solar power curtailment

wind converter reactive power

solar inverter reactive power, at node $j$ and time $t$ 


\section{Results and Discussion}

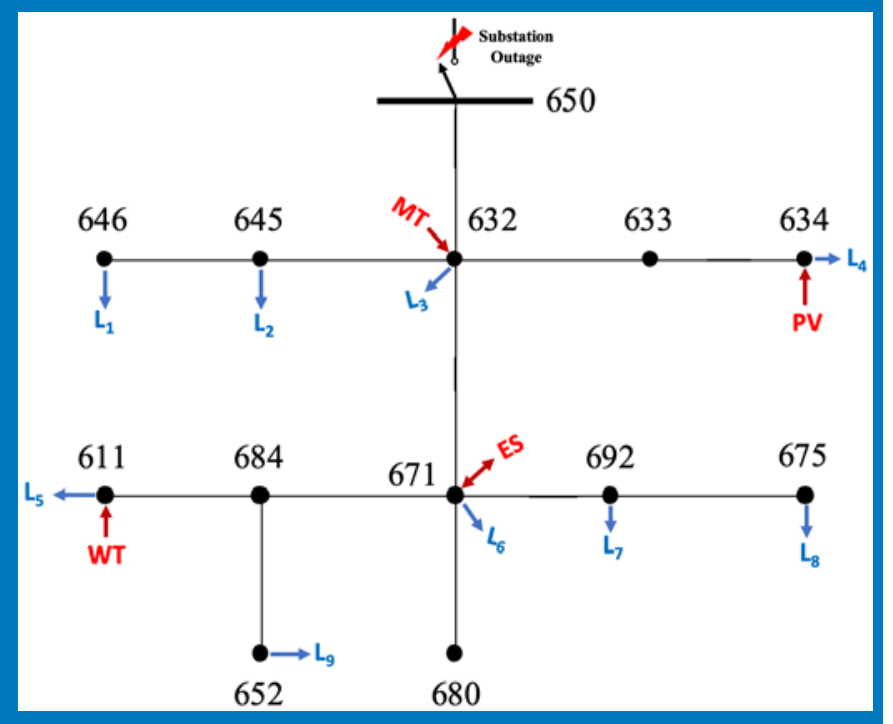

Modified IEEE 13-bus test feeder integrated with DERs and in islanded operation mode

\section{Simulation Details}

- We apply the proposed MPC-based service restoration method to the IEEE 13-bus distribution test system integrated with WT, PV, MT, and ES.

- We consider 9 spot loads $\left(L_{1}-L_{9}\right)$ with constant power load models.

- We assume an extreme event-caused substation outage occurred at 12:00.

- The utility outage time is set as 6 hours and the control step is $5 \mathrm{~min}$.

- We implemented the MPC using JuMP in Julia v1.5.

- The renewable power forecasting, data analytics and the MPC execution are performed in Python 3.7.

- The problem is MILP and solved by leveraging GLPK OS solver. 


\section{Results and Discussion}

System Parameters

\begin{tabular}{|c|c|c|c|}
\hline Parameter & Value & Parameter & Value \\
\hline$\omega$ & $\begin{array}{c}{[1.0,1.0,0.9,0.85,0.8,0.65} \\
0.45,0.4,0.3]\end{array}$ & $\mathrm{T}$ & $6 \mathrm{~h}$ \\
\hline$\alpha$ & 0.2 & $\Delta \mathrm{t}$ & $1 / 12$ \\
\hline$\beta$ & 0.2 & $\mathrm{P}_{\max }^{\mathrm{g}}$ & $300 \mathrm{~kW}$ \\
\hline$\psi$ & 100 & $\mathrm{Sg}^{\mathrm{g}}$ & $350 \mathrm{kVA}$ \\
\hline$\phi$ & 50 & $\mathrm{E}_{\max }^{\mathrm{g}, \mathrm{p}}$ & $1000 \mathrm{kWh}$ \\
\hline Pl,demand & $\begin{array}{c}{[115,85,49.75,200,85,199.75} \\
85,324,64] \mathrm{kW}\end{array}$ & $\mathrm{E}_{\max }^{\mathrm{g}, \mathrm{q}}$ & 750kvarh \\
\hline $\mathrm{Q}^{\mathrm{l}, \mathrm{demand}}$ & $\begin{array}{c}{[66,52,29,115,40,109,45,141,} \\
43] \text { kvar }\end{array}$ & $\mathrm{P}_{\max }^{\mathrm{es}, \mathrm{ch}}$ & $200 \mathrm{~kW}$ \\
\hline $\mathbf{t}$ & {$[1,2, \ldots, 72]$} & $\mathrm{P}_{\max }^{\mathrm{es}, \mathrm{dch}}$ & $200 \mathrm{~kW}$ \\
\hline $\begin{array}{l}\text { WT } \\
\text { rating }\end{array}$ & $150 \mathrm{~kW}$ & $\mathrm{~S}^{\mathrm{es}, \mathrm{inv}}$ & $250 \mathrm{kVA}$ \\
\hline PV rating & $300 \mathrm{~kW}$ & $\mathrm{SOC}_{0}^{\mathrm{es}}$ & $90 \%$ \\
\hline $\mathrm{C}^{\mathrm{es}}$ & $800 \mathrm{kWh}$ & $\mathrm{SOC}_{\min }^{\mathrm{es}}$ & $20 \%$ \\
\hline $\mathbf{L B}, \mathbf{U B}$ & $0.95,1.05 \mathrm{pu}$ & $\mathrm{SOC}_{\max }^{\mathrm{es}}$ & $100 \%$ \\
\hline$\eta^{\text {es,ch }}$ & $95 \%$ & $\eta^{\mathrm{es}, \mathrm{dch}}$ & $90 \%$ \\
\hline
\end{tabular}




\section{Results and Discussion Case Studies}

We discuss the simulation results obtained from our experiments based on the following 3 cases:

$\checkmark$ Case I: System operation without reserve

$\checkmark$ Case II: System operation with different levels of reserve requirements

$\checkmark$ Case III: System operation under renewable shortfall and over-generation 


\section{Results and Discussion \\ Case I - Operation w/o Reserve}

\section{Power dispatch and load restoration}

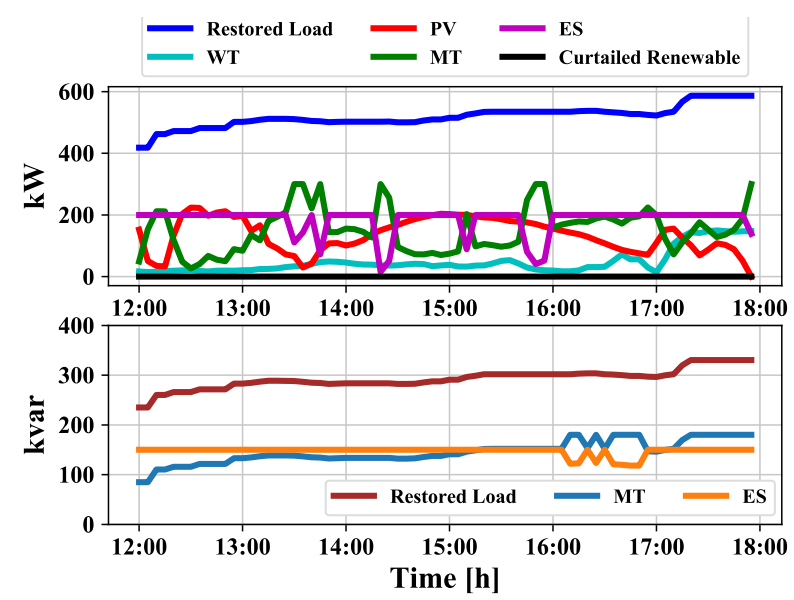

- The MPC operates the DERs interactively to restore the distribution system load

- The MT and ES operate complementary

- The MPC effectively utilized the renewables without curtailment

- We only set the MT and ES for reactive pow.
Individual restored loads

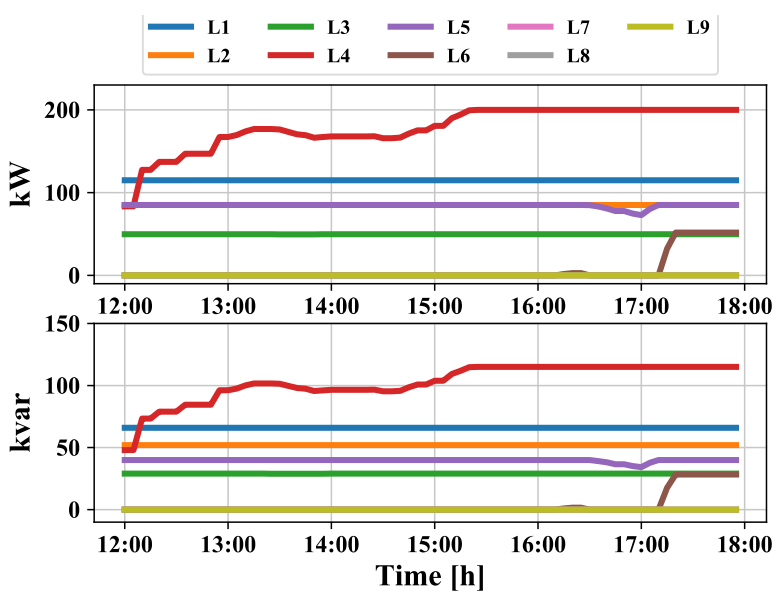

- The first 3 higher priority (critical) loads (L1 - L3) are picked up with their full demand (100\%) throughout the control horizon.

- The lowest priority loads (L7 - L9) were not served at all.
Nodal voltages

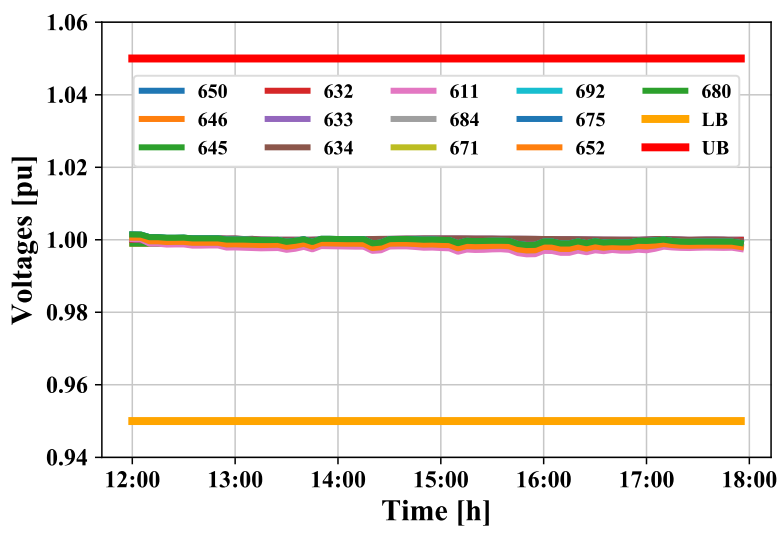

- The MPC is able to regulate the system node voltages well within the allowable ANSI's low-voltage distribution grid voltage range 0.95 to $1.05 \mathrm{pu}$. 


\section{Results and Discussion}

\section{Case II - Operation w/ Reserves}

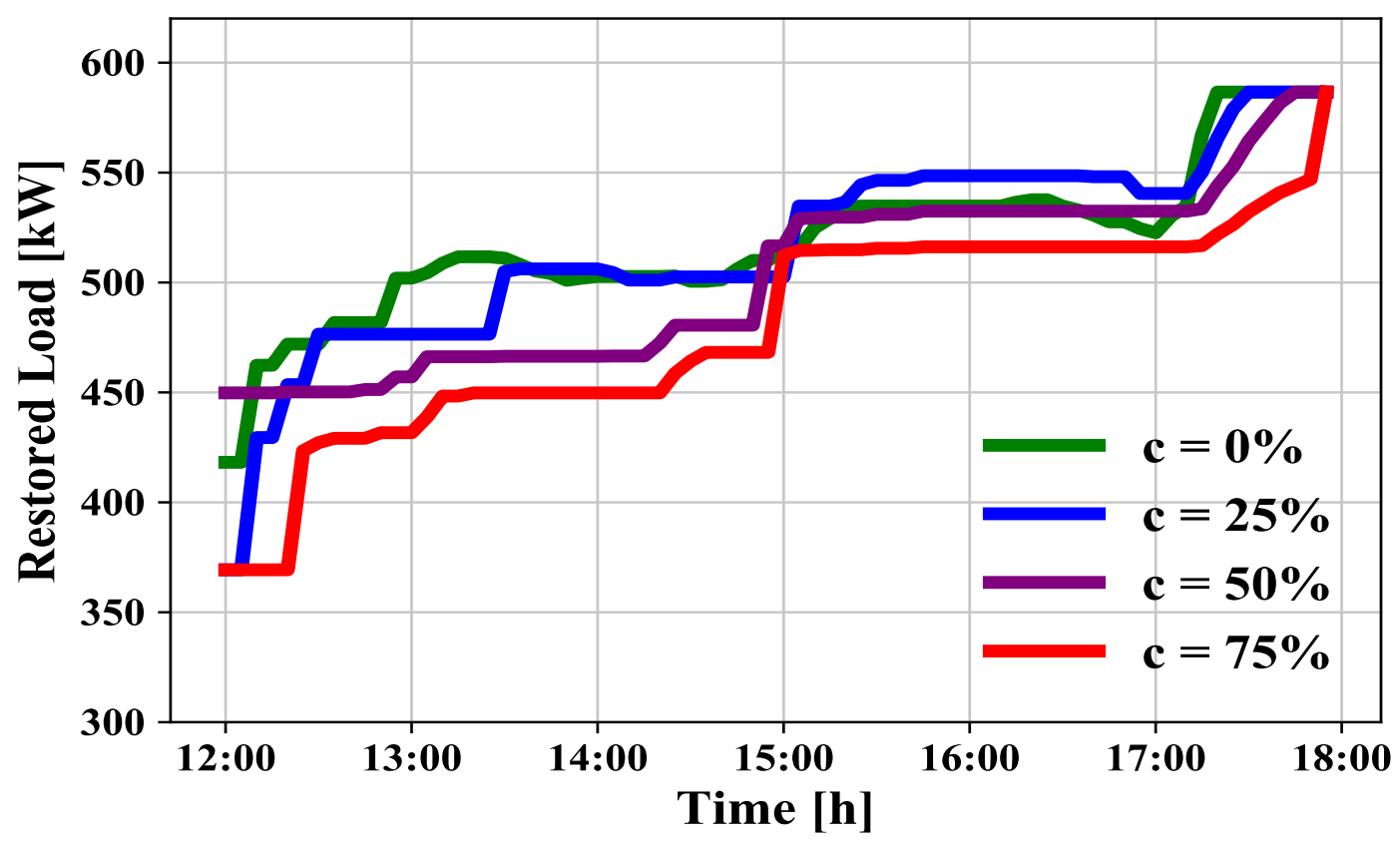

- Different levels of reserve requirement (from $c=0 \%$ up to $75 \%)$

$$
R_{t}=c \sum_{j \in N}\left(P_{j, t}^{w t}+P_{j, t}^{p v}\right)
$$

- As the system-wide reserve requirement increases, the aggregate restored load becomes more upward monotonic and there does not exist any shedding of previously restored load.

- The restoration curve with the highest value of reserve requirement $(\mathrm{c}=75 \%)$ restored the loads conservatively with consideration of monotonic increase as time evolves.

- This confirms the benefit of having reserve in the system to ensure the restoration is sustainable without shedding previously restored loads. 


\section{Results and Discussion}

\section{Case III - Operation under Renewable Shortfall and Surplus}
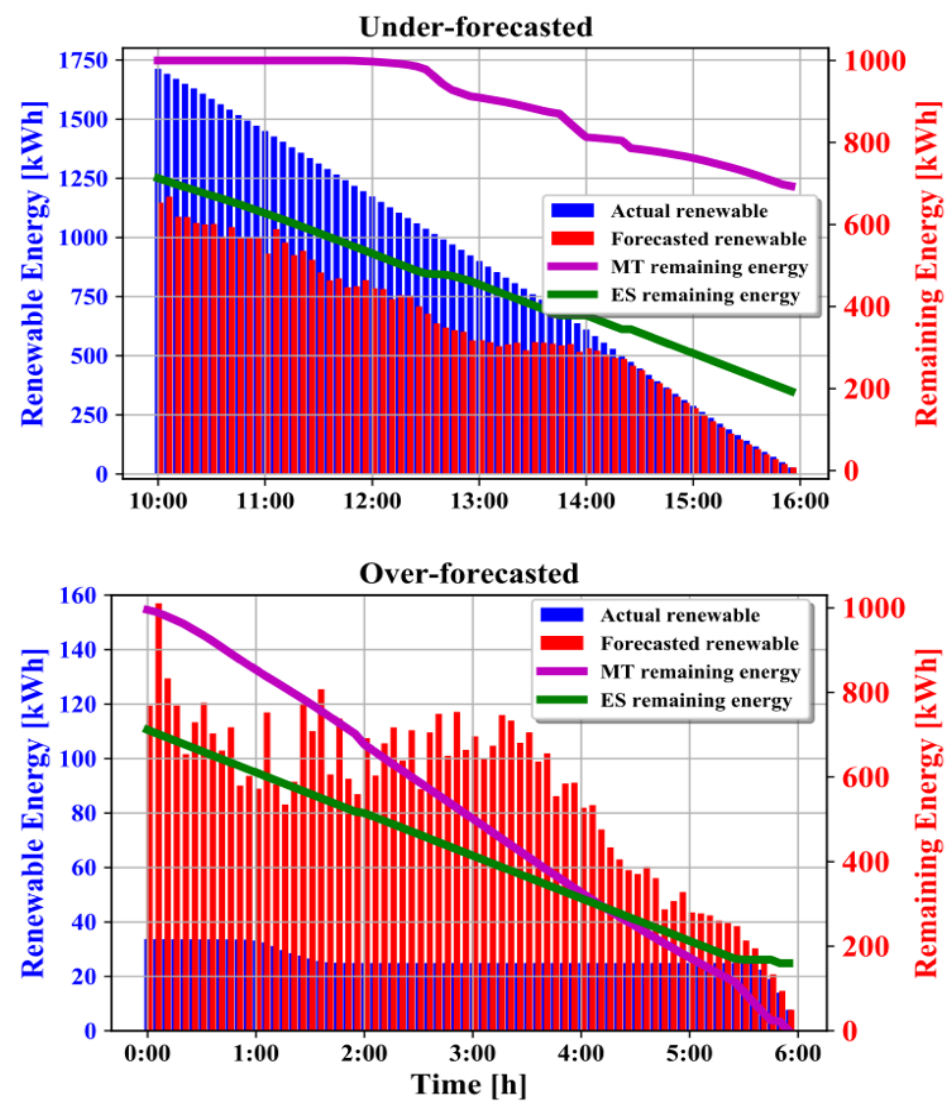

- With under-forecasted (forecast < actual) renewable (wind + solar) case the MPC does not utilize all the available fuel of the MT and the stored energy in the ES.

- This is due to the MPC initially expecting that the system has less renewables and but later on realizing that this is not true and prefers to use the available renewable instead of feeding the system from the MT and ES.

- During the over-forecasted (forecast $>$ actual) renewable case, the MPC uses all the available fuel of the MT (remaining energy = $0 \mathrm{kWh}$ at $\mathrm{t}=6: 00)$ and stored energy of the $\mathrm{ES}(\mathrm{min} \mathrm{SOC}=20 \%=$ $160 \mathrm{kWh}$ at $\mathrm{t}=6: 00)$.

- This is because initially the MPC expects the system has more renewable generation available but later on in realtime it realizes that this is not true and forced to feed more energy from the available MT and ES to restore more loads.

- This confirms the importance of MPC-based restoration approach in handling the uncertainty of renewables through realtime realization and decision adjustment. 
- Resiliency have become a vital property of critical systems and communities.

- Our findings reveal that deployment of DERs and

Conclusion and Future Work adopting robust distribution grid automations (such as automatic and critical service restoration algorithms) play significant roles in improving the resilience of power systems against extreme events.

- The current research findings and capabilities will continue in the next phases of our research such as consideration of more complex power networks and advanced stochastic formulations to address the uncertainty of service recovery time and renewables. 


\section{Thank you! \\ Questions?}

\section{www.nrel.gov}

NREL/PR-2C00-79530

This work was authored by the National Renewable Energy Laboratory, operated by Alliance for Sustainable Energy, LLC, for the U.S. Department of Energy (DOE) under Contract No. DE-AC36-08GO28308. Funding provided by the Improving Distribution System Resiliency via Deep Reinforcement Learning Project funded by the U.S. Department of Energy Office of Electricity Advanced Grid Modeling program. The views expressed in the article do not necessarily represent the views of the DOE or the U.S. Government. The U.S. Government retains and the publisher, by accepting the article for publication, acknowledges that the U.S. Government retains a nonexclusive, paid-up, irrevocable, worldwide license to publish or reproduce the published form of this work, or allow others to do so, for U.S. Government purposes.

\section{WNREL \\ Transforming ENERGY}

